\title{
Presentation and outcomes of patients with undifferentiated thyroid carcinoma: a national perspective
}

\author{
Zaid Al-Qurayshi ${ }^{1}$, Christopher Blake Sullivan ${ }^{1}$, Helmi Khadra ${ }^{2}$, Mohamed Shama $^{2}$, Grace S. Lee ${ }^{2}$, \\ Emad Kandil $^{2}$
}

${ }^{1}$ Department of Otolaryngology - Head \& Neck Surgery, University of Iowa Hospitals and Clinics, Iowa City, IA, USA; ${ }^{2}$ Department of Surgery, Tulane University School of Medicine, New Orleans, LA, USA

Contributions: (I) Conception and design: All author; (II) Administrative support: Z Al-Qurayshi, E Kandil; (III) Provision of study materials or patients: Z Al-Qurayshi, E Kandil; (IV) Collection and assembly of data: Z Al-Qurayshi, CB Sullivan, H Khadra, E Kandil; (V) Data analysis and interpretation: All authors; (VI) Manuscript writing: All authors; (VII) Final approval of manuscript: All authors.

Correspondence to: Emad Kandil, MD, MBA, FACS. Department of Surgery, Tulane School of Medicine, 1430 Tulane Ave, SL-22, New Orleans, LA 70112, USA. Email: ekandil@tulane.edu.

Background: Undifferentiated thyroid cancer (UTC) accounts for only 1-2\% of all thyroid cancers. UTC is one of the most aggressive solid tumors with high metastatic rates and mortality. The objectives of this study are to examine the characteristics of patients with UTC and their overall survival.

Methods: Retrospective analysis utilizing the National Cancer Database, 2004-2014 is performed. The study population included adults ( $\geq 18$ years) patients with UTC or differentiated thyroid carcinoma (DTC), which served as a reference group.

Results: A total of 1,870 UTC and 209,707 DTC patients were identified. The median follow-up time of UTC patients was 3.9 months (interquartile range: 1.6-9.0 months). When compared to DTC patients, patients with UTC were more likely to be $\geq 45$-year-old [OR: 48.62, 95\% CI: $(35.75,66.14), \mathrm{P}<0.001$ ], male [OR: 2.02, 95\% CI: (1.84, 2.22), P<0.001], and/or black [OR: 1.27, 95\% CI: (1.08, 1.49), P=0.004]. UTC patients were more likely to have Medicaid/Medicare or no insurance and treated in low-volume hospitals $(\mathrm{P}<0.001)$. Overall survival in patients with UTC was lower in patients older than 65 years [OR: 1.63, 95\% CI: (1.12, 2.38), P=0.011], with multiple comorbidities [OR: 1.65, 95\% CI: (1.02, 2.67), P=0.040] and/or presented with metastatic disease [OR: 1.93, 95\% CI: $(1.71,2.17), \mathrm{P}<0.001]$. Compared to thyroidectomy alone, patients without metastasis who received adjuvant radiotherapy and/or chemotherapy had a better overall survival $(\mathrm{P}<0.001$ each). In patients with metastatic disease, any intervention or combination of interventions other than thyroidectomy alone improved survival $(\mathrm{P}<0.05)$.

Conclusions: Older age, male, and/or black are associated with a higher prevalence of UTC compared to DTC. Although overall survival is poor in UTC, utilization of multi-modal treatment may improve survival.

Keywords: Epidemiology; undifferentiated thyroid carcinoma; differentiated thyroid carcinoma (DTC); metastasis; thyroid surgery

Submitted Dec 31, 2020. Accepted for publication Apr 14, 2021.

doi: $10.21037 /$ gs-20-927

View this article at: https://dx.doi.org/10.21037/gs-20-927

\section{Introduction}

By 2030, thyroid cancer is projected to become the $4^{\text {th }}$ most common cancer in the United States (1). Undifferentiated thyroid cancer (UTC), also known as anaplastic thyroid cancer, accounts for only 1-2\% of all thyroid cancers; however, it is, unfortunately, one of the most aggressive solid tumors with high metastatic rates and mortality $(2,3)$. Incidence rates of UTC have been stable over the previous four decades, but the median survival period of approximately four months has not changed in over fifty 
years (4-6). All patients with UTC are diagnosed as Stage IV according to the American Joint Commission on Cancer $(2,7)$. Given the course of rapid disease progression and poor prognosis, a multidisciplinary team of oncologists, radiation oncologist, surgeons, and palliative care providers should be involved in the treatment planning.

The American Thyroid Association (ATA) recommends aggressive surgical resection with radiation therapy in patients with local disease (2). Given the rapid progression of the disease, however, there is much heterogeneity with individual treatment patterns. Published studies are often retrospective studies limited by small sample size, due to the rare and aggressive nature of the disease. Molecular investigations have been ongoing to identify genes responsible for UTC, but targeted therapies are still in the development phase. The majority of patients are treated with conventional therapeutic interventions. Analyzing the effectiveness of therapies at a population level may provide additional insight on survival outcome. The objective of this study was to analyze the clinical characteristics and treatment outcomes of patients with UTC, compared to differentiated thyroid cancer (DTC) in the United States. We present the following article in accordance with the "STrengthening the Reporting of OBservational studies in Epidemiology" reporting checklist (available at https:// dx.doi.org/10.21037/gs-20-927).

\section{Methods}

A retrospective cohort analysis was performed utilizing the National Cancer Data Base (NCDB), 2004-2014 (8). The NCDB is a joint program of the Commission on Cancer of the American College of Surgeons (ACS) and the American Cancer Society (8). The ACS has executed a business associate agreement that includes a data use agreement with each of its Commission on Cancer accredited hospitals (8). The NCDB, established in 1989, is a nationwide, facilitybased, comprehensive clinical surveillance resource oncology data set that currently captures $70 \%$ of all newly diagnosed malignancies in the US annually (8). The NCDB is available by application to ACS. The NCDB includes de-identified publicly available data that do not meet the criteria of human subject research and the requirement of review by the institutional review board (8). The study was conducted in accordance with the Declaration of Helsinki (as revised in 2013).

The first objective of the study is to assess the demographic and clinical characteristics of patients who were diagnosed with UTC $v s$. DTC. The second objective is to assess factors associated with overall survival in patients with UTC.

The study population included adult patients (age $\geq 18$ years), who had UTC based on the International Classification of Diseases for Oncology third edition (ICD-O-3) codes: 8020, 8021, 8022; and patients with DTC based on ICD-O-3 codes: 8050, 8260, 8340, 8341, $8342,8343,8344,8330,8331,8332,8335,8450$.

Independent factors that were considered for their confounding effect included: age $(18-<45,45-<65$, $\geq 65$ ), gender, race (white, black, Hispanic, other), metastasis status, Charlson/Deyo comorbidity score as coded by the database $(0,1,2, \geq 3)$, type of treatment (thyroidectomy only, chemotherapy only, radiotherapy only, thyroidectomy \& chemotherapy, thyroidectomy \& radiotherapy, chemotherapy \& radiotherapy, thyroidectomy, chemotherapy, \& radiotherapy), hospital volume classification followed quartile classification based on the total number of thyroidectomies performed per year (low: $\leq 25$ th percentile, 1-16 thyroidectomies/year, intermediate: $>25$ th- $\leq 75$ th percentiles, 17-63 thyroidectomies/year, high: $>75$ th percentile, $\geq 64$ thyroidectomies/year), and type of insurance as coded in the database (none, private, Medicaid, Medicare, other governmental). All factors were checked for completeness. Any subject with a missing value for the above factors was excluded.

\section{Statistical analysis}

Chi-square test was used to compare the baseline characteristics of patients in relation to the thyroid cancer type (UTC $v s$. DTC). Factors with significant association were included in the multivariate logistic regression model to examine the characteristics of patients who had a higher prevalence of UTC vs. DTC. Kaplan-Meier curve and LogRank test were used to assess association of each independent risk factor with overall survival. Factors with a significant association with survival were considered potential confounders and were included in the multivariate Cox Hazard ratio (HR) model. Cox HR model was used to assess time interaction term and calculate adjusted HR and $95 \%$ confidence interval (95\% CI). Statistical significance was set as $\alpha=0.05$. All tests were two-sided. All statistical analyses were performed using SAS 9.4 (SAS Institute Inc., Cary, NC, USA.).

\section{Results}

A total of 1,870 patients with UTC and 209,707 patients 
Table 1 Descriptive statistics of the study population. National Cancer Database, 2004-2014

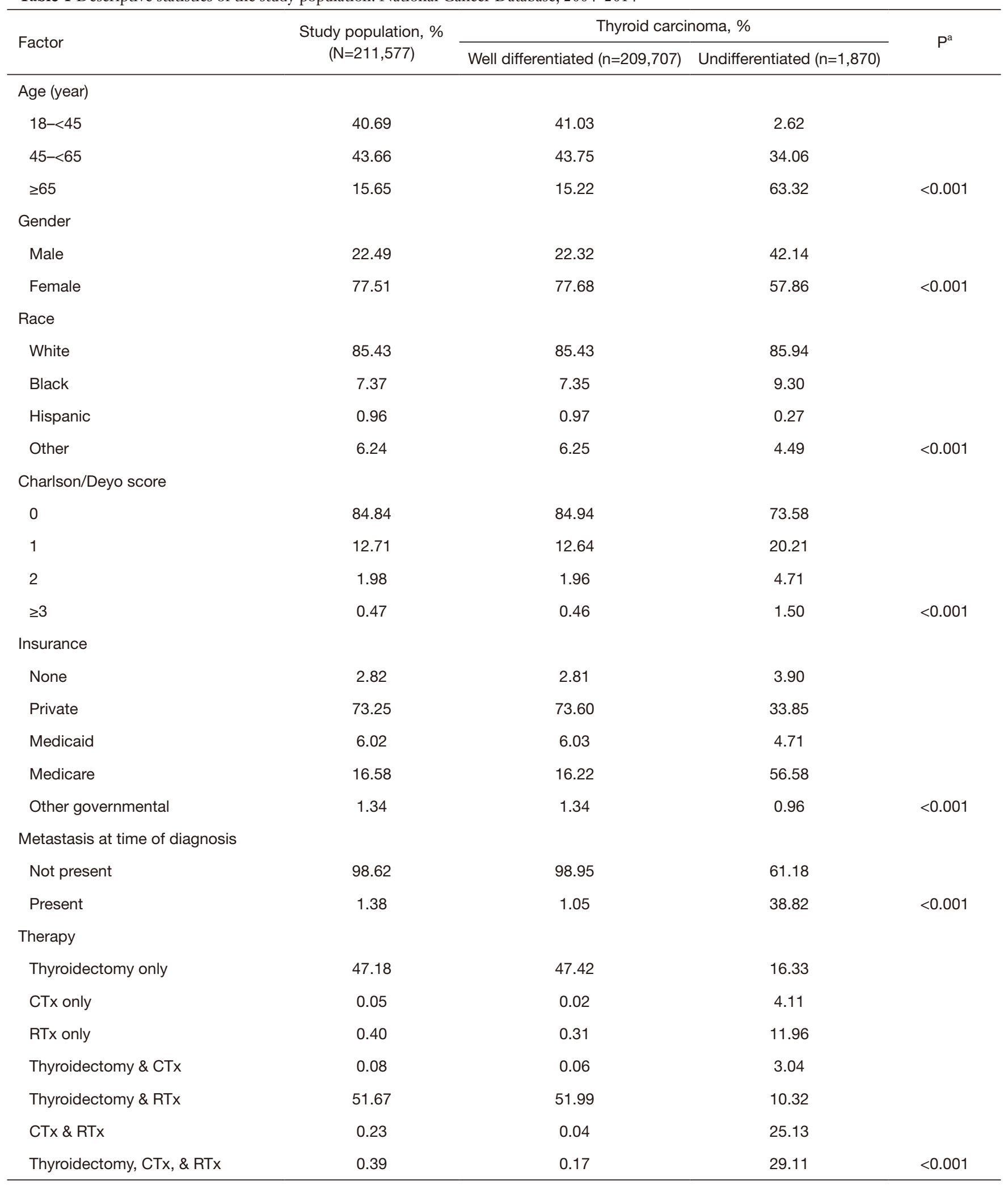

Table 1 (continued) 
Table 1 (continued)

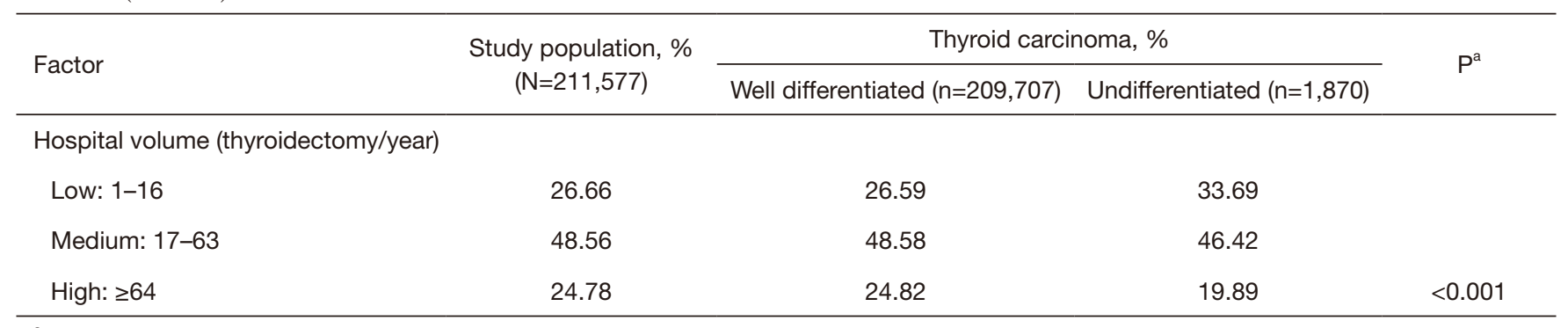

a, Chi-square test. CTx, chemotherapy; RTx, radiotherapy.

with DTC were included (Table 1). The mean age of patients with UTC was $69.0 \pm 12.2$ years, while the mean age of patients with DTC was $48.5 \pm 14.7$ years. The median follow-up period of UTC patients was 3.9 months (interquartile range: 1.6-9.0 months), and the median follow-up period of DTC was 60.2 months (interquartile range: $34.4-91.0$ months).

Compared to DTC, patients with UTC were more likely to be $\geq 45$ years old, male, of black racial background, and have multiple comorbidities $(\mathrm{P}<0.05$ each) (Table 2). UTC patients were most likely to have no insurance or Medicaid/ Medicare and likely to be managed in low-volume hospitals $(\mathrm{P}<0.05$ each $)$.

Overall survival in patients with UTC was lower in patients older than 65 years who have multiple comorbidities and presented with metastatic disease $(\mathrm{P}<0.05$ each) (Table 3). Compared to thyroidectomy alone, patients without metastasis who received thyroidectomy \& radiotherapy, or chemotherapy \& radiotherapy, or thyroidectomy, chemotherapy, \& radiotherapy had better overall survival $(\mathrm{P}<0.001$ each) (Figure 1). In patients with metastatic disease, any intervention or combination of interventions other than thyroidectomy alone improved survival $(\mathrm{P}<0.05$ each) (Figure 2).

\section{Discussion}

UTC is a rare form of thyroid cancer with aggressive local disease, high metastasis rates, and a very poor overall prognosis $(9,10)$. While UTC is responsible for only $1-2 \%$ of all thyroid cancers, the vast majority of thyroid cancerrelated deaths are due to UTC $(2,9)$. Currently, there is no optimal treatment algorithm for patients with UTC, but a combination of treatment modalities is often pursued (11). The goal of therapy is to prolong survival or palliate symptoms, as the cure rate is extremely low. The present study examines the characteristics of patients with UTC and their survival outcomes from a population level to provide epidemiologic and prognostic information.

The overall survival rate at two years was $27.6 \%$ in patients without metastasis and $8.01 \%$ with metastatic disease. These results are comparable to a study of 735 patients in the Surveillance, Epidemiology, and End Results database, which showed a 2-year overall survival of 10.7 (12). While the 2-year overall survival remains poor, patients without metastasis who underwent tri-modal therapy including surgery, had the best overall survival. While the ATA recommends multimodal therapy of surgery, radiation therapy, and chemotherapy in patients with resectable stage IVA/IVB disease (2), the disease may change rapidly and limit the treatment options (13). In the current study, even patients with Stage IVB/IVC (presence of metastasis) had improved overall survival at 2 years with tri-modal therapy compared to monotherapy. If surgery is chosen, aggressive macroscopic oncologic resection has shown to improve outcomes compared to palliative resection only (9). Unfortunately, the only surgical option for some UTC patients may be a tracheotomy to prevent upper airway obstruction, but surgical resection should strongly be considered with adjuvant therapy in appropriate patients.

A substantial number of patients will not be able to undergo surgery due to the advanced stage of their disease or due to quality of life preferences. Of non-surgical treatments, radiation therapy alone was the least effective as a monotherapy in patients with or without metastasis. In patients without metastasis, chemotherapy alone or chemotherapy\& radiation therapy had better 2-year overall survival than radiation therapy alone. Patients with metastasis treated with chemotherapy \& radiation therapy had improved 2-year overall survival compared to either chemotherapy or radiation therapy alone. Currently, there is no standard chemotherapy regimen for UTC patients, 
Table 2 Characteristics of patients with undifferentiated thyroid carcinoma as compared to patients with well-differentiated thyroid carcinoma $(\mathrm{N}=211,577)$

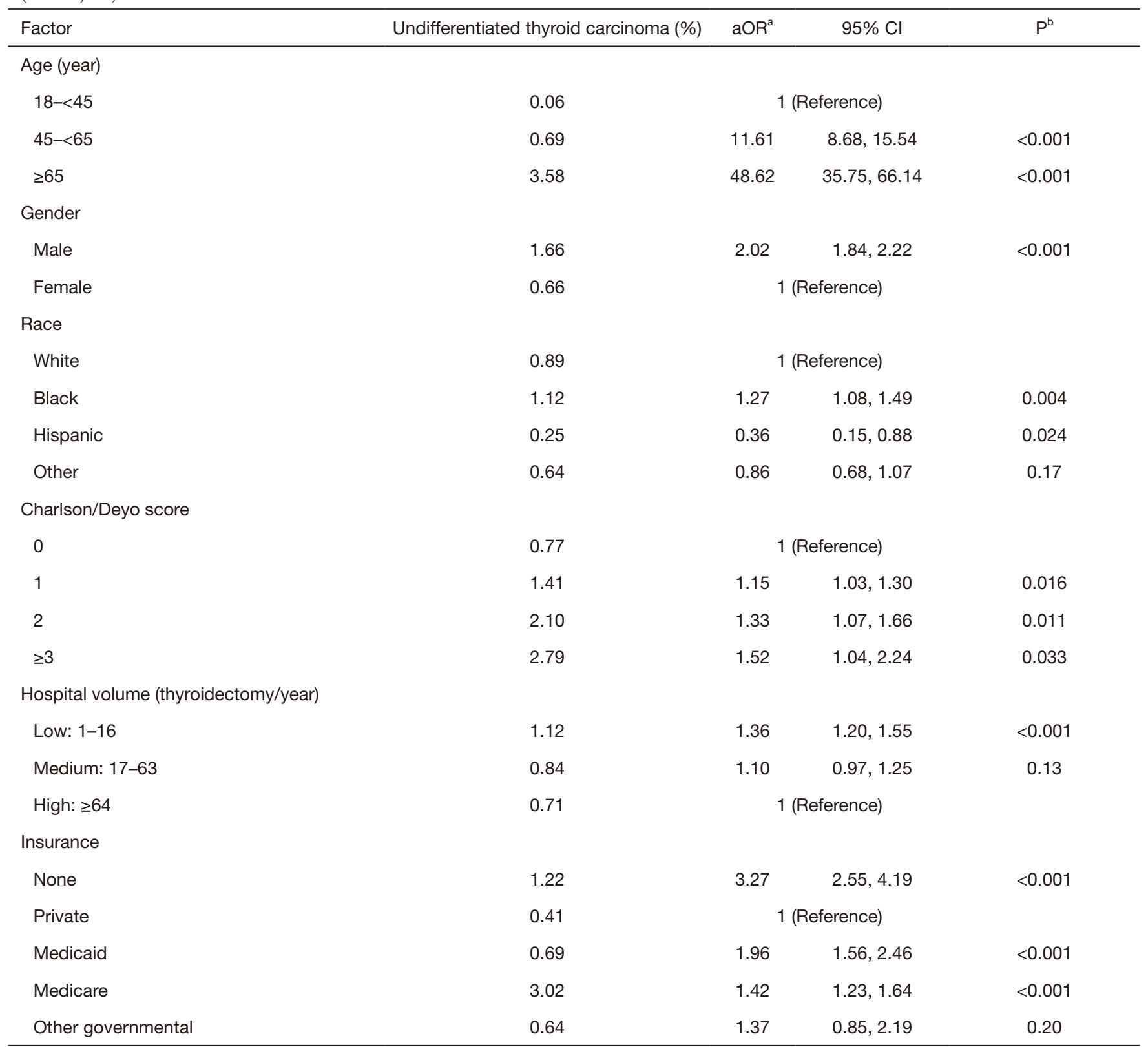

a, the model includes: age, gender, race, Charlson/Deyo comorbidity score, hospital volume, and insurance. ${ }^{\mathrm{b}}$, multivariate logistic regression model. aOR, adjusted odds ratio; $\mathrm{Cl}$, confidence interval.

and they often receive systemic therapy as part of a clinical trial $(14,15)$. Chemotherapy regimens have included doxorubicin, cisplatin, docetaxel, and/or paclitaxel $(14,16)$. Despite many variations in the chemotherapy regimen, our results show that patients should be offered chemotherapy with radiation therapy if their functional status allows it. Lowe et al. showed that the combination of chemotherapy and radiation therapy improved median survival to 220 days compared to radiation therapy alone at 58.5 days and chemotherapy alone at 137 days. Patients who are candidates for a combination therapy should be counseled about the potential severe toxicity, given the location of the primary tumor and higher doses of radiation that are often employed along with systemic therapy (17). Ongoing, 
Table 3 The association of selected demographic and clinical factors with overall survival in patients with undifferentiated thyroid carcinoma $(\mathrm{n}=1,870)$

\begin{tabular}{|c|c|c|c|c|c|}
\hline Factor & 1-year survival (\%) & 2-year survival (\%) & $\mathrm{aHR} \mathrm{R}^{\mathrm{a}}$ & $95 \% \mathrm{Cl}$ & $\mathrm{P}^{\mathrm{b}}$ \\
\hline $18-<45$ & 97.96 & 21.66 & \multicolumn{2}{|c|}{1 (Reference) } & \\
\hline $45-<65$ & 99.84 & 26.61 & 1.32 & $0.93,1.89$ & 0.12 \\
\hline$\geq 65$ & 99.66 & 15.88 & 1.63 & $1.12,2.38$ & 0.011 \\
\hline White & 99.81 & 20.88 & \multicolumn{2}{|c|}{1 (Reference) } & \\
\hline Black & 99.43 & 15.45 & 1.08 & $0.90,1.30$ & 0.40 \\
\hline Hispanic & 80.00 & 0.00 & 1.70 & $0.55,5.29$ & 0.36 \\
\hline Other & 97.62 & 15.04 & 1.12 & $0.87,1.46$ & 0.38 \\
\hline Present & 99.59 & 8.01 & 1.93 & $1.71,2.17$ & $<0.001$ \\
\hline \multicolumn{6}{|l|}{ Charlson/Deyo score } \\
\hline 0 & 99.71 & 22.21 & \multicolumn{2}{|c|}{1 (Reference) } & \\
\hline 1 & 99.74 & 15.42 & 1.34 & $1.17,1.53$ & $<0.001$ \\
\hline 2 & 98.86 & 10.41 & 1.69 & $1.32,2.17$ & $<0.001$ \\
\hline$\geq 3$ & 96.43 & 3.57 & 1.65 & $1.02,2.67$ & 0.040 \\
\hline \multicolumn{6}{|l|}{ Insurance } \\
\hline None & 97.26 & 25.90 & 1.11 & $0.82,1.49$ & 0.51 \\
\hline Thyroidectomy only & 99.49 & 82.06 & \multicolumn{2}{|c|}{1 (Reference) } & \\
\hline CTx only & 94.44 & 83.33 & 0.57 & $0.32,1.03$ & 0.06 \\
\hline RTx only & 98.73 & 79.75 & 1.16 & $0.88,1.54$ & 0.29 \\
\hline Thyroidectomy \& CTx & 100.00 & 96.43 & 0.73 & $0.48,1.12$ & 0.15 \\
\hline Thyroidectomy \& RTx & 99.21 & 95.28 & 0.46 & $0.36,0.60$ & $<0.001$ \\
\hline$C T x \& R T x$ & 99.47 & 94.71 & 0.61 & $0.48,0.77$ & $<0.001$ \\
\hline Thyroidectomy, CTx, \& RTx & 99.72 & 99.45 & 0.34 & $0.27,0.44$ & $<0.001$ \\
\hline \multicolumn{6}{|c|}{ Therapy (patients with metastasis) } \\
\hline Thyroidectomy only & 98.39 & 69.35 & \multicolumn{2}{|c|}{1 (Reference) } & \\
\hline CTx only & 97.87 & 82.98 & 0.55 & $0.36,0.82$ & 0.003 \\
\hline
\end{tabular}

Table 3 (continued) 
Table 3 (continued)

\begin{tabular}{lcccc}
\hline Factor & 1-year survival (\%) & 2-year survival (\%) & aHR $^{\mathrm{a}}$ & \multicolumn{1}{c}{$95 \% \mathrm{Cl}^{\mathrm{b}}$} \\
\hline RTx only & 99.09 & 67.27 & 0.68 & $0.48,0.95$ \\
Thyroidectomy \& CTx & 100.00 & 95.00 & 0.24 & $0.14,0.42$ \\
Thyroidectomy \& RTx & 97.22 & 88.89 & 0.26 & $0.17,0.41$ \\
CTx \& RTx & 99.52 & 93.74 & 0.29 & $0.21,0.42$ \\
Thyroidectomy, CTx, \& RTx & 98.98 & 96.94 & 0.17 & $0.11,0.27$ \\
\hline
\end{tabular}

${ }^{a}$, the multivariate model controls for: age, race, metastasis status, Charlson/Deyo score, therapy status, and insurance. ${ }^{\mathrm{b}}$, multivariate cox hazard ratio model. aHR, adjusted hazard ratio; $\mathrm{Cl}$, confidence interval; CTx, chemotherapy; RTx, radiotherapy.

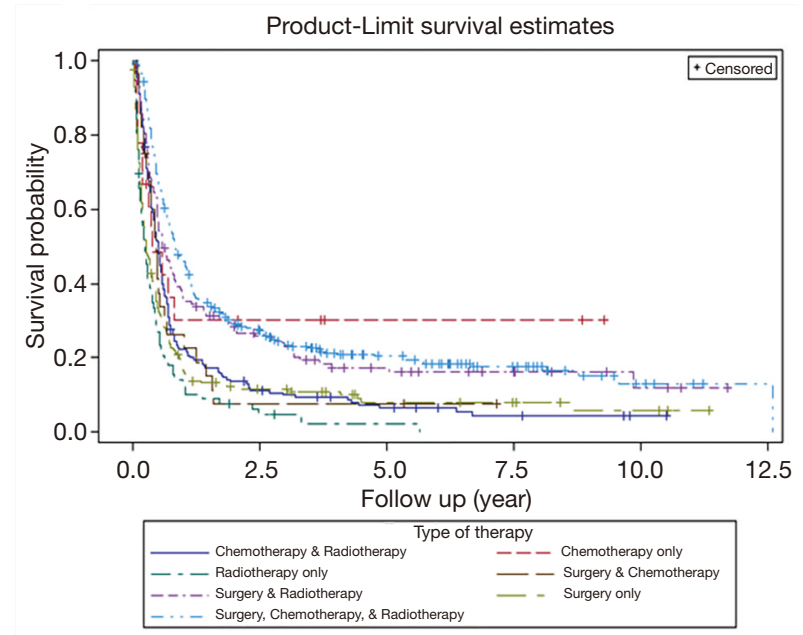

Figure 1 Overall survival of patients diagnosed with undifferentiated thyroid carcinoma without distant metastasis based on the treatment modality.

targeted clinical trials will be essential to further elucidate the role of non-surgical treatment in this terminal disease.

Racial characteristics of patients with UTC are not well-described in the literature. A previous retrospective analysis by Roche et al. showed that non-white patients had worse overall survival for UTC (18). We found that black Americans were more likely to have UTC compared to DTC. While not statistically significant, non-white patients showed a trend for worse overall survival compared to white patients. Furthermore, non-white patients are less likely to receive any treatment (18). These findings are consistent with head and neck cancer as a whole, as age-adjusted incidence in black Americans has increased, overall survival has decreased compared to the whites. Treatment regimens in black Americans are more variable compared to the regimens in white patients (19). Yu et al. found that black Americans

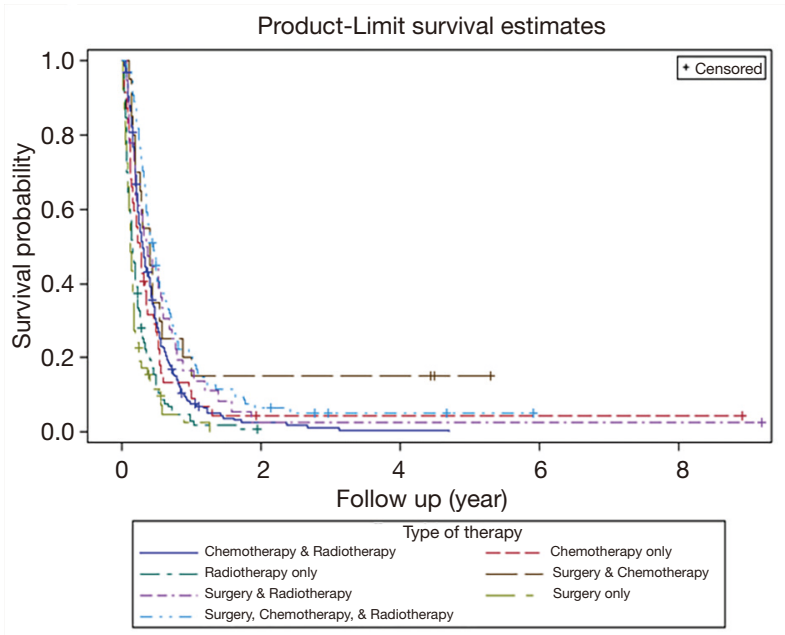

Figure 2 Overall survival of patients diagnosed with undifferentiated thyroid carcinoma with distant metastasis based on the treatment modality.

had the largest increase in thyroid cancer among racial groups from 1992-2004 (20). Further studies are needed to elucidate this racial disparity, which is likely multifactorial.

This retrospective study has multiple limitations. The patients in the present study were extracted from the $\mathrm{NCDB}$, which has the potential for coding misclassification. Additionally, these patients are only from Commission on Cancer accredited hospitals, which may result in selection bias (8). Causality cannot be inferred as the results are analyzed at a population level. UTC patients only had 3.9 months of median follow-up compared to 60.2 in patients with DTC, which is indicative of the grave prognosis. With respect to surgery, the database does not describe the extent of surgical resection. Despite these inherent limitations, we provide a population-level overview of survival outcomes of patients with UTC. 


\section{Conclusions}

This is one of the largest population-level studies of patients with UTC to provide epidemiologic and prognostic level data. We found that tri-modal therapy improves short-term survival, but overall survival continues to be dismal. Patients with metastasis who undergo non-surgical management have better overall survival with chemotherapy and radiation therapy than either monotherapy alone. Elderly patients, males, and black Americans have a higher prevalence of UTC.

\section{Acknowledgments}

This study was selected for the Surgical Forum presentation at the American College of Surgeons Clinical Congress, October 22-26, 2017in San Diego, CA.

Funding: Al-Qurayshi is supported by National Institutes of Health - Institutional National Research Award: T32 (\#5T32DC000040).

\section{Footnote}

Reporting Checklist: The authors have completed the STROBE reporting checklist. Available at https://dx.doi. org/10.21037/gs-20-927

Data Sharing Statement: Available at https://dx.doi. org/10.21037/gs-20-927

Conflicts of Interest: All authors have completed the ICMJE uniform disclosure form (available at https://dx.doi. org/10.21037/gs-20-927). Emad Kandil serves as an Editorin-Chief of Gland Surgery. The other authors have no conflicts of interest to declare.

Ethical Statement: The authors are accountable for all aspects of the work in ensuring that questions related to the accuracy or integrity of any part of the work are appropriately investigated and resolved. The NCDB includes de-identified publicly available data that do not meet the criteria of human subject research and the requirement of review by the institutional review board. The study was conducted in accordance with the Declaration of Helsinki (as revised in 2013).

Open Access Statement: This is an Open Access article distributed in accordance with the Creative Commons
Attribution-NonCommercial-NoDerivs 4.0 International License (CC BY-NC-ND 4.0), which permits the noncommercial replication and distribution of the article with the strict proviso that no changes or edits are made and the original work is properly cited (including links to both the formal publication through the relevant DOI and the license). See: https://creativecommons.org/licenses/by-nc-nd/4.0/.

\section{References}

1. La Vecchia C, Malvezzi M, Bosetti C, et al. Thyroid cancer mortality and incidence: a global overview. Int $\mathrm{J}$ Cancer 2015;136:2187-95.

2. Smallridge RC, Ain KB, Asa SL, et al. American Thyroid Association Guidelines for Management of Patients with Anaplastic Thyroid Cancer. Thyroid 2012;22:1104-39.

3. Saini S, Tulla K, Maker AV, et al. Therapeutic advances inanaplastic thyroid cancer: a current perspective. Mol Cancer 2018;17:154.

4. Olson E, Wintheiser G, Wolfe KM, et al. Epidemiology of Thyroid Cancer: A Review of the National Cancer Database, 2000-2013. Cureus 2019;11:e4127.

5. Davies L, Welch HG. Increasing incidence of thyroid cancer in the United States, 1973-2002. JAMA 2006;295:2164-7.

6. Smallridge RC, Marlow LA, Copland JA. Anaplastic thyroid cancer: molecular pathogenesis and emerging therapies. Endocr Relat Cancer 2009;16:17-44.

7. American Joint Commission on Cancer. Available online: https://cancerstaging.org/Pages/default.aspx. Published 2016.

8. American college of surgeons. National cancer data base. Available online: https://www.facs.org/quality-programs/ cancer/ncdb

9. Molinaro E, Romei C, Biagini A, et al. Anaplastic thyroid carcinoma: from clinicopathology to genetics and advanced therapies. Nat Rev Endocrinol 2017;13:644-60.

10. Glaser SM, Mandish SF, Gill BS, et al. Anaplastic thyroid cancer: Prognostic factors, patterns of care, and overall survival. Head Neck 2016;38 Suppl 1:E2083-90.

11. Giuffrida D, Gharib H. Anaplastic thyroid carcinoma: current diagnosis and treatment. Ann Oncol 2000;11:1083-9.

12. Huang NS, Shi X, Lei BW, et al. An Update of the Appropriate Treatment Strategies in Anaplastic Thyroid Cancer: A Population-Based Study of 735 Patients. Int J Endocrinol 2019;2019:8428547.

13. Kebebew E, Greenspan FS, Clark OH, et al. Anaplastic 
thyroid carcinoma. Treatment outcome and prognostic factors. Cancer 2005;103:1330-5.

14. Keutgen XM, Sadowski SM, Kebebew E. Management of anaplastic thyroid cancer. Gland Surg 2015;4:44-51.

15. Lowe NM, Loughran S, Slevin NJ, et al. Anaplastic Thyroid Cancer: The Addition of Systemic Chemotherapy to Radiotherapy Led to an Observed Improvement in Survival--A Single Centre Experience and Review of the Literature. ScientificWorldJournal 2014;2014:674583.

16. Seto A, Sugitani I, Toda K, et al. Chemotherapy for anaplastic thyroid cancer using docetaxel and cisplatin: report of eight cases. Surg Today 2015;45:221-6.

17. Cabanillas ME, Zafereo M, Gunn GB, et al. Anaplastic

Cite this article as: Al-Qurayshi Z, Sullivan CB, Khadra H, Shama M, Lee GS, Kandil E. Presentation and outcomes of patients with undifferentiated thyroid carcinoma: a national perspective. Gland Surg 2021;10(6):1971-1979. doi: 10.21037/gs-20927
Thyroid Carcinoma: Treatment in the Age of Molecular Targeted Therapy. J Oncol Pract 2016;12:511-8.

18. Roche AM, Fedewa SA, Shi LL, et al. Treatment and survival vary by race/ethnicity in patients with anaplastic thyroid cancer. Cancer 2018;124:1780-90.

19. Daraei P, Moore CE. Racial Disparity Among the Head and Neck Cancer Population. J Cancer Educ 2015;30:546-51.

20. Yu GP, Li JCL, Branovan D, et al. Thyroid cancer incidence and survival in the national cancer institute surveillance, epidemiology, and end results race/ethnicity groups. Thyroid 2010;20:465-73. 